\title{
Recuperación de apatita desde relaves en columna de flotación
}

\author{
Luis I. Valderrama ${ }^{(1) \star}$, Jaime M. Tapia(2), Osvaldo E. Gómez ${ }^{(3)}$ y Mario E. Santander ${ }^{(1)}$ \\ (1) Universidad de Atacama. Departamento de Ingeniería en Metalurgia. Copiapó, Chile. \\ (correo-e: luis.valderrama@uda.cl; mario.santader@uda.cl) \\ (2) Universidad Arturo Prat. Facultad de Ingeniería y Arquitectura. Iquique - Chile. (correo-e: jtapia@unap.cl) \\ (3) Unidad de Geología, Compañía de Acero del pacifico, La Serena - Chile. (correo-e: oegomez@cmp.cl)
}

* Autor a quien debe ser dirigida la correspondência.

Recibido Jun. 7, 2019; Aceptado Ago. 5, 2019; Versión final Sep. 6, 2019, Publicado Feb. 2020

\section{Resumen}

Se ha estudiado y analizado la flotación de apatita contenida en el relave producido en el proceso de concentración magnética de la Planta de Pellet de la Compañía Minera del Pacífico. La actividad minera produce anualmente millones de toneladas de relaves generados por el tratamiento de minerales, los cuales se descargan en forma de pulpa en los tranques. En los últimos años se han realizado varios estudios tendientes a recuperar minerales de valor contenidos en tales relaves. Es por ello que se ejecutaron pruebas de flotación en una mini columna de flotación, donde se analizaron las siguientes variables: altura de espuma, flujo de aire, alimentación y agua de lavado, con dosis de colector y espumante constante a pH 9 . Se concluye que es posible recuperar el $75,3 \%$ de $\mathrm{P}_{2} \mathrm{O}_{5}$ contenido en el relave con una ley de $21,8 \%$ en una sola etapa, usando un flujo de aire de $1,4 \mathrm{~cm} / \mathrm{s}$, agua de lavado $0,41 \mathrm{~cm} / \mathrm{s}$, de alimentación $1,4 \mathrm{~cm} / \mathrm{s}$ y una altura de espuma de $15 \mathrm{~cm}$, usando $400 \mathrm{~g} / \mathrm{t}$ de colector, $400 \mathrm{~g} / \mathrm{t}$ de dispersante y $10 \mathrm{~g} / \mathrm{t}$ de espumante.

Palabras clave: apatita; recuperación de minerales; relave de hierro; concentración magnética

\section{Recovery of apatite from tailings in flotation column}

\begin{abstract}
Flotation of apatite contained in the tailings produced in the magnetic concentration process of the Pellet Plant of the Pacific Mining Company has been studied and analyzed. The mining activity annually produces millions of tons of tailings generated by the treatment of minerals, which are discharged in the form of pulp in dams. In recent years there have been several studies aimed to the recovering of valuable minerals contained in such tailings. For this reason, flotation tests were performed in a mini flotation column, where the following variables were analyzed: froth height, air flow, feed and wash water, with collector and frother dose constant at $\mathrm{pH} 9$. It is concluded that it is possible to recover $75.3 \%$ of $\mathrm{P}_{2} \mathrm{O}_{5}$ contained in the tailings with a $21.8 \%$ grade in a single stage, using an air flow of $1.4 \mathrm{~cm} / \mathrm{s}$, washing water flow of $0.41 \mathrm{~cm} / \mathrm{s}$, feeding flow of $1.4 \mathrm{~cm} / \mathrm{s}$ and froth height of $15 \mathrm{~cm}$; using $400 \mathrm{~g} / \mathrm{t}$ of collector, $400 \mathrm{~g} / \mathrm{t}$ of dispersant and $10 \mathrm{~g} / \mathrm{t}$ of frother agent.
\end{abstract}

Keywords: apatite; mineral recovery; flotation, iron tailings; magnetic concentration 


\section{INTRODUCCIÓN}

La actividad minera produce anualmente millones de toneladas de relaves, situación que en los últimos años ha adquirido gran relevancia, porque se deben cumplir una serie de requerimientos de seguridad destinados a la protección de las personas y el medio ambiente. Se debe tener presente, que estos pasivos ambientales pertenecerán siempre a la empresa que los produjo. En la mayoría de los casos, estos relaves se descargan en tranques o embalses, donde los sólidos y los fluidos se separan por gravedad (Edraki, et al., 2014; Wang, et al., 2014). Su almacenamiento puede producir diversos daños ambientales, afectando recursos hídricos, biológicos, atmosféricos, socio-económicos y visuales. Si los relaves no son adecuadamente controlados, pueden producir drenaje ácido, contaminación del suelo, contaminación de las aguas y, eventualmente, la ruptura del tranque (Falagán, et al., 2017; Liang y Elias., 2010; Wolkersdorfer y Bowell, 2004). Nuestro país es el primer productor mundial de cobre con 5.800 .000 t/año, primer productor de yodo 18.000 t/año, segundo productor mundial de litio 16.000 t/año y segundo productor mundial de molibdeno 61.000 t/año. Esta gran producción de materias primas genera grandes volúmenes de relaves. Un estudio realizado por Sernageomin en el año 2017 (Sernageomin, 2017) sobre depósitos de relaves, indica que en la Región de Atacama, la Comuna de Copiapó posee 84 depósitos, que contienen una gran cantidad de pirita, magnetita, oro, plata, cobre, titanio, cuarzo y tierras raras.

El tratamiento de relaves se considera como una alternativa rentable cuando contienen cantidades relativamente altas de minerales de valor. Más aún, dado que los relaves no implican costos de extracción, chancado, molienda y procesamiento, su retratamiento permite generar un flujo de caja positivo. En ese mismo sentido, en Copiapó-Chile la Planta Magnetita, de la Compañía Minera del Pacífico, procesó 24 millones 940 mil toneladas métricas de relaves de la Compañía Contractual Minera Candelaria, con una ley promedio de hierro magnético alimentado de 9,89\%, produciendo 2.120 .000 toneladas de concentrado con una ley de Fe magnético de 63,17\% (Memoria Anual CAP, 2017). La planta M. A. Matta de ENAMI, tiene un tonelaje de relaves almacenado cercano a las 30.000 .000 toneladas y contiene magnetita, pirita, ilmenita, rutilo, calcopirita, oro y plata, así como también elementos de tierras raras. Por tal razón, resulta interesante la producción de concentrado de pirita mediante flotación a partir de estos relaves, haciendo que su beneficio técnico-económico sea bastante atractivo. De igual manera, en Estados Unidos, los relaves de la mina Pea Ridge de hierro, que operó durante 38 años, están siendo reprocesando para recuperar tierras raras Grauch, et al., (2010). Similarmente, en Chile los depósitos de relaves y ripios de las plantas de ENAMI (Valderrama, et al., 2003), presentan contenidos de tierras raras bastante elevados.

En este contexto, la búsqueda de alternativas para el manejo apropiado y sostenible de relaves ha culminado con estudios muy interesantes. Así, en investigaciones realizadas por Benzaazoua, et al., (2008) los relaves se utilizan como rellenos; de igual manera, otra investigación (Choi, et al., 2009) indican que pueden usarse como coberturas mediante las técnicas de solidificación/estabilización. Por otra parte, la flotación se usó para eliminar los sulfuros de los relaves, esta permite reducir sustancialmente el volumen de aguas ácidas (Broadhurst, et al., 2015; Mbamba, et al., 2012). Además de la flotación, se utiliza la concentración magnética y la concentración por gravedad para reprocesar estos relaves, produciendo concentrados de alto valor (Leistner et al., 2016; Bruckard y McCallum 2007). La recuperación de oro contenido en relaves se ha estudiado y aplicado con éxito en varios países, como Sudáfrica, República Dominicana, Canadá y Chile, (Durance, et al, 2010; Valderrama et al., 2011).

El desarrollo de tecnología para recuperar minerales de valor contenidos en los relaves ha sido estudiada por Oliveira et al., (2011) que investigó la flotación de fosfato de un relave con un contenido de 9,52\% $\mathrm{P}_{2} \mathrm{O}_{5}$, usando como colector uno sintético y aceite de arroz, obteniendo concentrado de $29,4 \% \mathrm{P}_{2} \mathrm{O}_{5}$ con una recuperación de 46,2\%; este relave es producido por una planta que produce fertilizante fosfatados en Araxá Brasil. Alsafasfeh y Alagha (2017) estudiaron recuperar $\mathrm{P}_{2} \mathrm{O}_{5}$ de relave producido por una planta de fosfato. Se realizaron pruebas de flotación en una celda Denver D-12, y se analizó el porcentaje de sólido, pH de la pulpa y el tiempo de flotación, usando silicato de sodio como y oleato de sodio. Los resultados obtenidos muestran un concentrado con ley entre $21,57 \%$ y $28,4 \%$ con recuperación de $73 \%$.

En el año 2017 Chile importó 347.885 toneladas de fertilizante para la agricultura por un valor por tonelada de US $\$ 397$, lo que indica que nuestro país requiere una gran cantidad de fosforo para mejorar la productividad de sus suelos. Con base a lo expuesto, este trabajo tiene como objetivo recuperar las apatitas contenidas en los relaves generados de la concentración magnética producidos por la Compañía de Acero del Pacífico, con ley de $\mathrm{P}_{2} \mathrm{O}_{5}$ por sobre $28 \%$ para la producción de fertilizantes.

\section{MATERIALES Y MÉTODOS}

La muestra de relave utilizada en este trabajo, se obtuvo de la Compañía de Acero del Pacífico, del embalse de relave el Trigo, que es producto de la concentración magnética de la planta El Romeral, de la Región de 
Coquimbo, Chile. Este embalse cuenta con $25 \mathrm{MMt}$, con una granulometría de $80 \%$ bajo 74 micrómetros. Así, la extracción de las muestras se realizó mediante un muestreo sistemático tendiente a cubrir la máxima superficie, por lo cual se empleó una perforadora con tornillo helicoidal rotatorio y una excavadora. Las muestras fueron clasificadas, homogenizadas y cuarteadas para ser caracterizada química-mineralógica, granulométrica y pruebas de flotación (Herrera, 2018). El análisis granulométrico se realizó deslamando en la malla 37 micrómetros y el material retenido sobre la malla se secó, homogenizó y analizo granulométricamente en una batería de abertura 210, 149, 105, 74, 53, 44, 37, -37 micrómetros. Paralelamente, el peso específico fue determinado por el método del picnómetro.

\section{Caracterización de la muestra de relave}

La composición química de la muestra se realizó por el método volumétrico del dicromato de potasio para el hierro, el fosforo por absorción molecular, el magnesio, calcio y aluminio por absorción atómica, el sodio y el potasio por fotometría de llama y la sílice por gravimetría. También fue usado un espectrómetro de fluorescencia de rayos X. El análisis mineralógico cuantitativo se realizó por Qemscan sobre briquetas transparente-pulidas, con tamaño de partícula que incluía material grueso $(+125 \mu \mathrm{m})$ y fino $(-44 \mu \mathrm{m})$.

\section{Pruebas de flotación}

Las pruebas de flotación se ejecutaron en una columna convencional de diámetro de $2,54 \mathrm{~cm}$ y una altura de $180 \mathrm{~cm}$. La inyección del aire se realizó mediante un difusor de acero poroso (porosidad entre 2 a $7 \mu \mathrm{m}$ ) que permite la formación de un gran número de pequeñas burbujas de diámetro relativamente uniforme (100 m), mientras que en el extremo superior se agregó el agua de lavado. Mediante flujometros se reguló los caudales de aire, alimentación y agua.

El concentrado se colectó en un recipiente de fondo inclinado, situado en la parte superior de la columna, donde se añadió agua para diluir y facilitar su escurrimiento. La interfase pulpa-espuma se controló en forma visual porque los módulos de la columna eran de vidrio transparente, lo que permitió visualizar la altura de la espuma durante toda la prueba. Después de alcanzado el estado estacionario de la columna, se colectaron muestras desde los flujos de concentrado, relave y alimentación, por un tiempo de 20 minutos. Las muestras colectadas se filtraron y secaron en estufas por 24 horas a temperaturas de $60{ }^{\circ} \mathrm{C}$, posteriormente se disgregaron, pesaron, homogenizaron y cuartearon, en cuarteador tipo Jones, siendo luego refinadas y enviadas para análisis químicos.

\section{RESULTADOS Y DISCUSION}

En la tabla 1, que muestra los resultados de los análisis químicos realizados al relave, se puede observar que la ley de $\mathrm{P}_{2} \mathrm{O}_{5}$ es de $2,66 \%$, de hierro total de $7,81 \%$, y un contenido de sílice bastante elevado de $41,4 \%$. Con dicho material se realizaron las pruebas de flotación en columna. De igual manera, el análisis mineralógico cuantitativo realizado al relave, indicando los distintos minerales que componen las muestras, se presentan en la Tabla 2. En dicha tabla se observa que la calcopirita es el único sulfuro de cobre presente en el relave, en un porcentaje de $0,1 \%$.

Tabla 1: Composición química del relave

\begin{tabular}{|l|l|l|l|l|l|l|l|l|}
\hline Relave & $\% \mathrm{Fe}$ & $\% \mathrm{P}_{2} \mathrm{O}_{5}$ & $\% \mathrm{MgO}$ & $\% \mathrm{CaO}$ & $\% \mathrm{Al}_{2} \mathrm{O}_{3}$ & $\% \mathrm{~K}_{2} \mathrm{O}$ & $\% \mathrm{Na}_{2} \mathrm{O}$ & $\% \mathrm{SiO}_{2}$ \\
\hline Porcentaje & 7,81 & 2,66 & 6,70 & 1,1 & 7,20 & 0,43 & 1,5 & 41,4 \\
\hline
\end{tabular}

Tabla 2: Minerales presentes en el relave

\begin{tabular}{|l|c|l|c|}
\hline Mineral & Porcentaje & Mineral & Porcentaje \\
\hline FeO $/$ Hidróxido & 30,7 & Piroxeno & 2,3 \\
\hline Calcopirita & 0,1 & Epidota & 3,0 \\
\hline Pirita & 2,6 & Clorita & 6,8 \\
\hline Cuarzo & 3,1 & Micas & 2,3 \\
\hline Feldespatos & 18,5 & Apatito & 3,4 \\
\hline Anfíbol & 19,9 & Otros & 6,8 \\
\hline
\end{tabular}


Los diferentes silicatos que constituyen la ganga (cuarzo, feldespatos, anfíbola, epídota, clorita y micas) representan el $53,6 \%$ del relave, mientras que los minerales con contenido de hierro corresponden aproximadamente el $33.3 \%$, y se encuentran presentes como magnetita $\left(\mathrm{Fe}_{3} \mathrm{O}_{4}\right)$, pirita $\left(\mathrm{FeS}_{2}\right)$, limonita $\left(\mathrm{FeO}(\mathrm{OH}) \cdot \mathrm{nH}_{2} \mathrm{O}\right)$ y siderita $\left(\mathrm{FeCO}_{3}\right)$. El contenido de apatita $\mathrm{Ca}_{5}\left(\mathrm{PO}_{4}\right)_{3}(\mathrm{~F}, \mathrm{Cl}, \mathrm{OH})$ alcanza al $3,4 \%$. El peso específico determinado por el método del picnómetro entregó un valor de $2,8 \mathrm{~kg} / \mathrm{L}$.

En la figura 1 se presentan los resultados del análisis granulométrico y la distribución en porcentaje de $\mathrm{P}_{2} \mathrm{O}_{5}$ por cada fracción granulométrica. Se observa que el $46,7 \%$ de las partículas tiene un tamaño menor a 37 micrómetros y que el $42,2 \%$ de $\mathrm{P}_{2} \mathrm{O}_{5}$ del total del fosfato se encuentra contenido bajo este mismo tamaño granulométrico. Se debe hacer notar que el alto porcentaje de material menor a 53 micrómetros, que corresponde al $67,3 \%$, podría anticipar una limitada recuperación y selectividad en la pruebas de flotación en columna, puesto que este proceso opera adecuadamente a granulometrías entre los 105 y 74 micrómetros, esto fue demostrado en la flotación de varios minerales por Laskowski (1989).

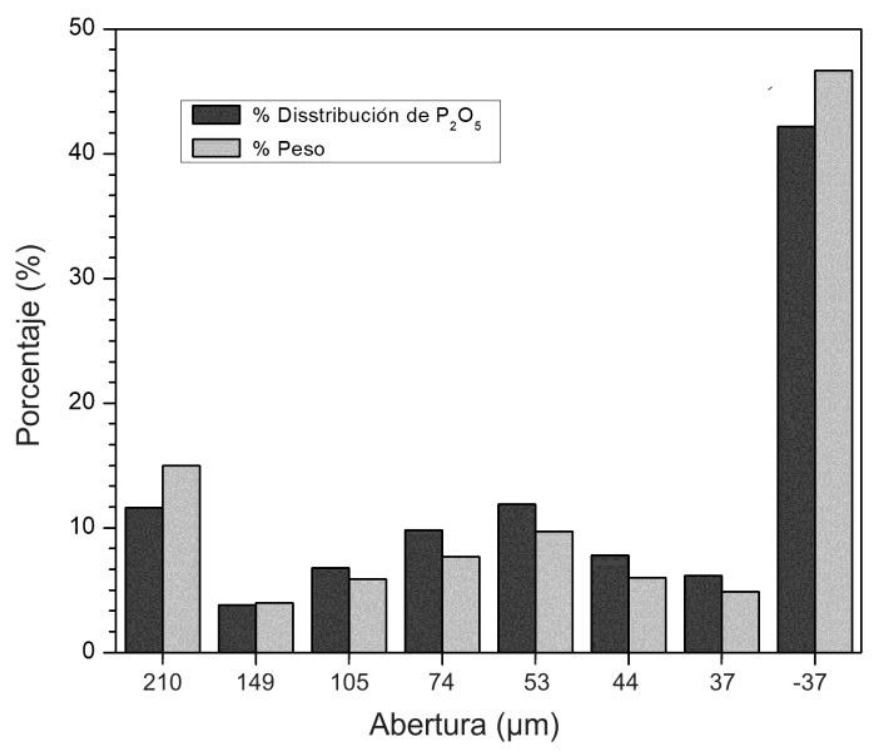

Fig. 1: Distribución granulométrica y de $\mathrm{P}_{2} \mathrm{O}_{5}$ por tamaño

Inicialmente la pulpa se reguló a pH 9, agregando posteriormente el colector Atrac 2600 (reactivo sintético de AkzoNobel), en una dosis de $400 \mathrm{~g} / \mathrm{t}$, el dispersante silicato de sodio (400 g/t) y espumante MIBC (10 g/t), y acondicionándola por $10 \mathrm{~min}$; estas dosificaciones y $\mathrm{pH}$ fueron optimizados en pruebas preliminares realizadas en celdas convencionales de flotación a escala de laboratorio (Oliva, 2018). La alimentación a la columna se realizó mediante una bomba peristáltica, .cuyas velocidades superficiales de alimentación de pulpa fluctuaron en el intervalo de 1,0,1,2,1,5 y $1,6 \mathrm{~cm} / \mathrm{s}$, con un porcentaje de sólido de $28 \%$. El relave se descargó de la columna usando otra bomba peristáltica.

La prueba de flotación se inició encendiendo la bomba de agua de lavado, luego la bomba de relave y posteriormente la bomba de alimentación. Con la bomba de relave se reguló la altura pulpa-espuma para mantener un bias positivo. Alcanzado el estado estacionario, se inició la toma de muestra de concentrado y relave. Estas muestras colectadas se decantaron, filtraron y secaron a $80^{\circ} \mathrm{C}$, para luego ser pesadas, disgregadas, cuarteadas y pulverizadas, para su posterior análisis químico.

En la figura 2 se presentan los resultados de recuperación y ley de $\mathrm{P}_{2} \mathrm{O}_{5}$ obtenidos en las pruebas de flotación en columna, usando relave como alimentación. La figura muestra la variación de la ley y recuperación en función del flujo de aire inyectado a la columna. De ella se deduce que al elevar la velocidad superficial del aire aumenta la recuperación de $\mathrm{P}_{2} \mathrm{O}_{5}$ en el concentrado, ya que esta incrementa el hold-up (fracción volumétrica ocupada por el aire en cualquier punto de la columna), el tamaño de la burbuja adherida al sólido y también la colisión burbuja-partícula, Robinstein, (1995). En este caso, se estudiaron cuatro velocidades superficiales de aire (intervalos usados industrialmente) 1,2, 1,3, 1,4 y 1,5 cm/s. Esta tendencia continúa hasta el valor de velocidad superficial de $1,4 \mathrm{~cm} / \mathrm{s}$, mientras que para valores más altos la recuperación disminuye, posiblemente debido a la coalescencia de las burbujas, lo que hace disminuir la eficiencia de la flotación. Tal tendencia también fue informada por Tariq et al., (2013). La recuperación metalúrgica, o eficiencia, es el cuociente porcentual entre la masa de $\mathrm{P}_{2} \mathrm{O}_{5}$ recuperada en el concentrado y la masa de $\mathrm{P}_{2} \mathrm{O}_{5}$ alimentada a la flotación 


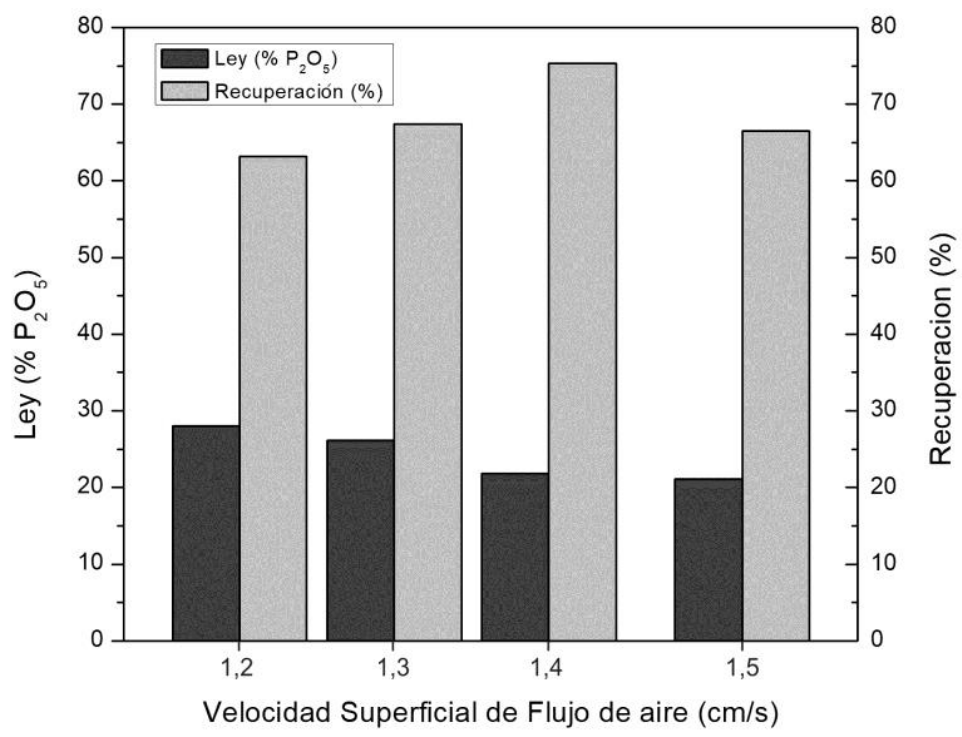

Fig. 2: Recuperación y ley de $\mathrm{P}_{2} \mathrm{O}_{5}$ en función de las velocidades de aire

Un segundo grupo de pruebas de flotación se ejecutó para estudiar el efecto de la altura de espuma sobre la recuperación y la ley de $\mathrm{P}_{2} \mathrm{O}_{5}$ en el concentrado. Las otras variables se mantuvieron constantes en $1,4 \mathrm{~cm} / \mathrm{s}$ de velocidad superficial de alimentación, $0,41 \mathrm{~cm} / \mathrm{s}$ de agua de lavado y $1,4 \mathrm{~cm} / \mathrm{s}$ de aire; valores que se usan en columnas de flotación a nivel industrial. En la figura 3 se presentan los resultados obtenidos al variar la altura de espuma entre 10, 15, 20 y $25 \mathrm{~cm}$, en función de la recuperación y ley de $\mathrm{P}_{2} \mathrm{O}_{5}$. Como se aprecia en la figura, a medida que la altura de espuma aumenta, la recuperación disminuye, de $68,4 \%$ hasta $54,3 \%$, mientras que la ley $\mathrm{P}_{2} \mathrm{O}_{5}$ permanece casi constante, en valores de $25,1 \%$ a $25,7 \%$. Para la altura de espuma de $10 \mathrm{~cm}$, se obtiene una recuperación de $68,4 \%$ con una ley de $25,9 \%$ de $\mathrm{P}_{2} \mathrm{O}_{5}$. En función de la altura de espuma, las partículas hidrofóbicas se dirigen hacia el concentrado, mientras que la ganga se drena hacia la pulpa. De esta manera, a medida que la altura de espuma aumenta la recuperación de fosfato disminuye, porque las burbujas de aire se encuentren menos mineralizadas por el agua de lavado. Este comportamiento ha sido observado anteriormente por Hunter et al., (2008).

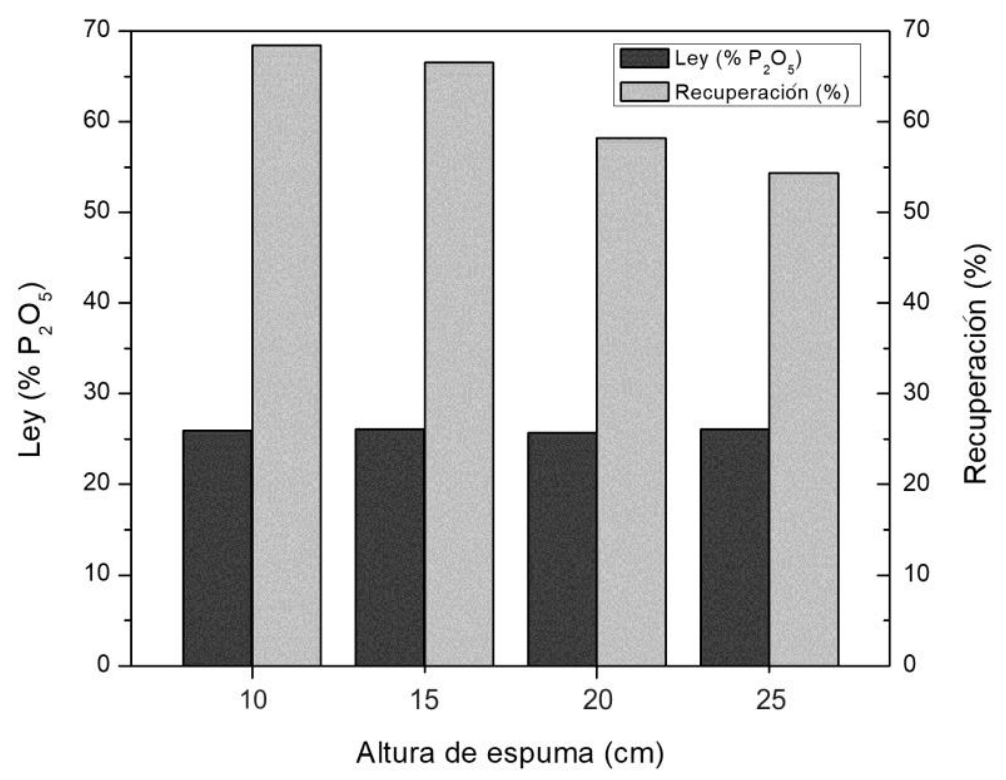

Fig. 2: Recuperación y ley de $\mathrm{P}_{2} \mathrm{O}_{5}$ en función de las velocidades de aire 
La variación de la ley y recuperación de fosfato en función del flujo de agua de lavado se presenta en la Figura 4. Las demás variables operacionales se mantuvieron constantes en un valor de velocidad superficial de alimentación de $1,4 \mathrm{~cm} / \mathrm{s}$, velocidad de aire en $1,4 \mathrm{~cm} / \mathrm{s}$ y una altura de espuma de $15 \mathrm{~cm}$. Tal como se indicó precedentemente, dichos valores de velocidad superficial son los utilizados a nivel industrial. En la figura se presentan los resultados obtenidos para velocidades superficiales de agua de lavado de $0,16,0,26,0,33$ y $0,41 \mathrm{~cm} / \mathrm{s}$. Se observa en la figura que al aumentar la velocidad superficial del agua de lavado la recuperación disminuye desde $82,0 \%$ hasta $68,0 \%$, mientras que la ley de $\mathrm{P}_{2} \mathrm{O}_{5}$ en el concentrado aumenta desde $12,9 \%$ hasta $25,0 \%$. Se debe indicar que cuando la recuperación metalúrgica disminuye y la ley de fosfato en el concentrado se incrementa la recuperación en peso también disminuye, desde 17,6\% a 7,7\%. La función del agua de lavado es drenar las partículas finas y mixtas de ganga arrastradas a la fase de espuma; por tales razones, se obtienen concentrados con contenidos de la especie útil mayor; disminuyendo la recuperación y la estabilidad de la columna. En concordancia con lo anterior, es posible mencionar que estudios realizados por Von Holt y Franzidis (1994) y Abdel-Zaher (2008) han tenido resultados similares para el agua de lavado en columnas de flotación con rocas fosfatadas.

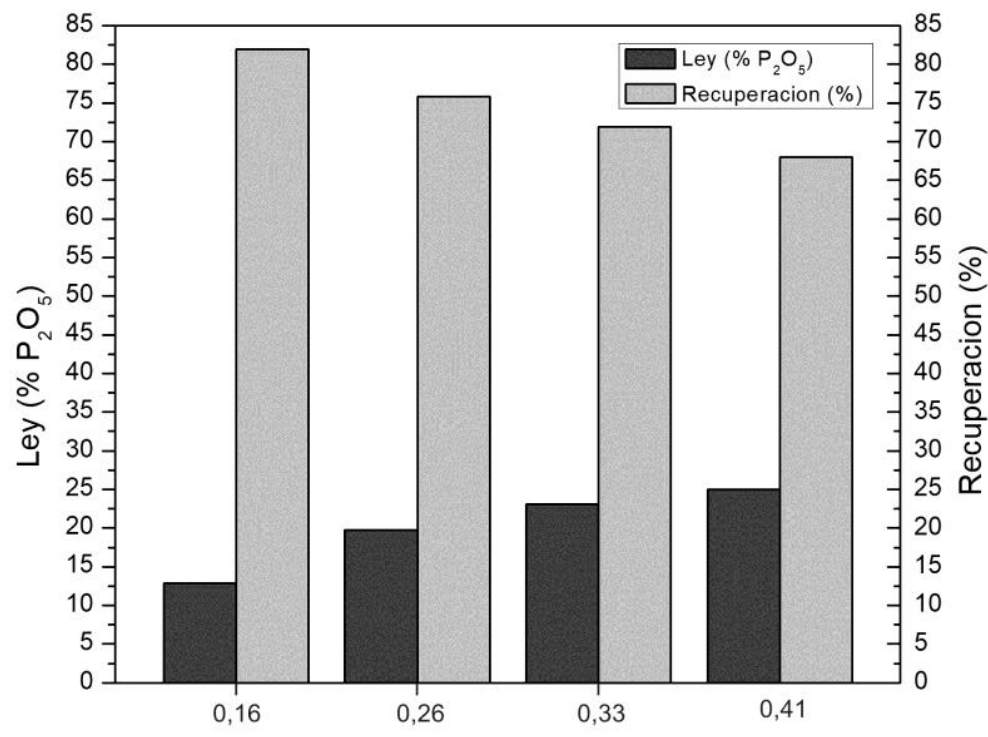

Velocidad Superficial de Agua de Lavado $(\mathrm{cm} / \mathrm{s})$

Fig. 4: Recuperación y ley de $\mathrm{P}_{2} \mathrm{O}_{5}$ en función de la velocidad de agua de lavado

La figura 5 muestra la variación de la ley y recuperación de $\mathrm{P}_{2} \mathrm{O}_{5}$ en función de la velocidad superficial de alimentación de pulpa. Las demás variables operacionales se mantuvieron constantes en un valor de velocidad superficial de aire de $1,4 \mathrm{~cm} / \mathrm{s}$, velocidad superficial de agua de lavado de $0,41 \mathrm{~cm} / \mathrm{s}$ y una altura de espuma de $15 \mathrm{~cm}$. Las velocidades superficiales de alimentación de pulpa fluctuaron en el intervalo de 1,0, $1,2,1,5$ y $1,6 \mathrm{~cm} / \mathrm{s}$. En esta figura se observa que a medida que se incrementa la velocidad superficial de alimentación de pulpa a la columna, la recuperación de $\mathrm{P}_{2} \mathrm{O}_{5}$ en el concentrado disminuye desde un valor de $76,9 \%$, para una velocidad superficial de $1,0 \mathrm{~cm} / \mathrm{s}$ a una recuperación de $41,3 \%$ para una velocidad superficial de $1,6 \mathrm{~cm} / \mathrm{s}$, mientras que en el mismo intervalo de velocidad superficial la ley de fosfato se incrementa desde $19,8 \%$ de $\mathrm{P}_{2} \mathrm{O}_{5}$ a $29,1 \%$ de $\mathrm{P}_{2} \mathrm{O}_{5}$; esto indica que la eficiencia de adhesión burbujas-partículas disminuye a altas velocidades superficiales de alimentación, lo que resulta en una menor recuperación de $\mathrm{P}_{2} \mathrm{O}_{5}$, esto se explica porque la estabilidad de los agregados partícula-burbuja disminuye y es explicada por Schulze (1977).

La figura 5 también indica que existe un valor máximo en la recuperación de $\mathrm{P}_{2} \mathrm{O}_{5}$ alcanzando un valor de $76,9 \%$, para la velocidad superficial de alimentación de 1,0 cm/s. Resultados similares sobre la flotación de fosfatos han sido informados por los investigadores Al-Fariss et al., (2017). Dado que la ley de $\mathrm{P}_{2} \mathrm{O}_{5}$ que se requiere para comercializar estos concentrados para la producción de fertilizantes es de $28 \%$ de $\mathrm{P}_{2} \mathrm{O}_{5}$, para asegurar una alta recuperación y alcanzar las leyes requeridas de fosfato en el concentrado, se debe considerar en trabajos futuros pruebas de flotación (cleaner) para limpiar el concentrado producido y también al relave, someterlo a una nueva etapa de flotación (etapa scavenger), de esta manera obtener una la ley de fosfato requerida y mantener una recuperación aceptable (Robinstein, 1995; Eisele y Kawatra, 1999). 


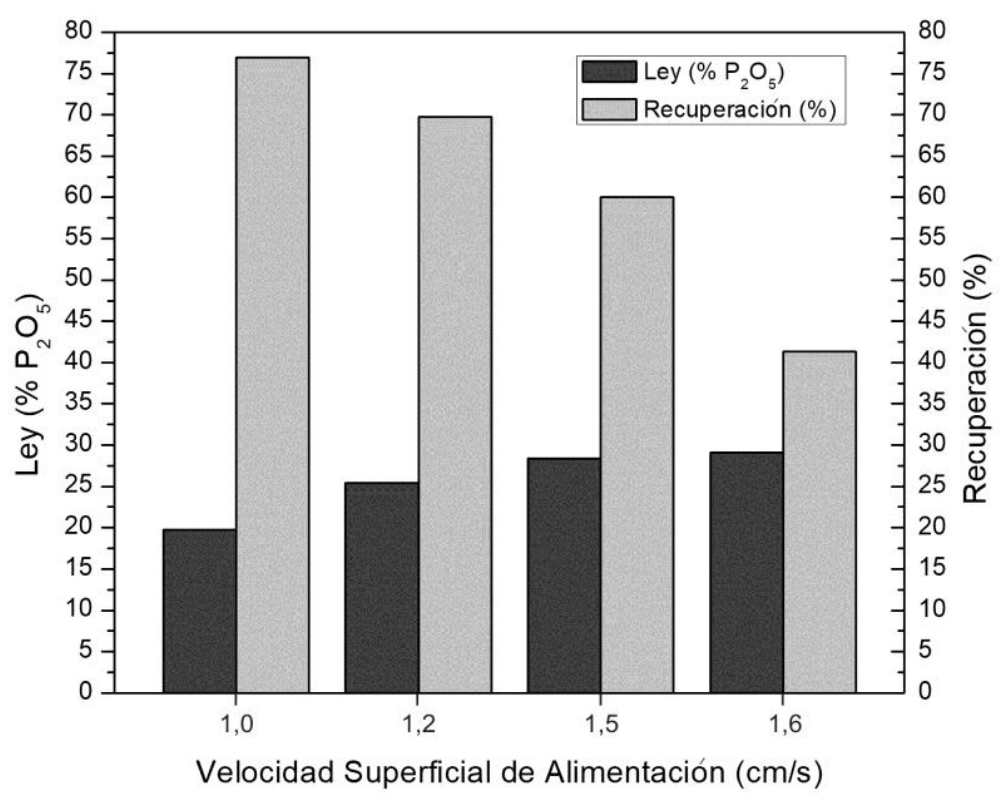

Fig. 5: Recuperación y ley de $\mathrm{P}_{2} \mathrm{O}_{5}$ en función de la velocidad de alimentación

\section{CONCLUSIONES}

Los resultados obtenidos en esta investigación, usando una columna como una etapa rougher de flotación directa, permiten establecer las siguientes conclusiones: 1 ) Mediante los análisis químicos se determinó que el relave contiene $2,66 \%$ de $\mathrm{P}_{2} \mathrm{O}_{5}$ y que el $42,2 \%$ de las partículas tiene un tamaño menor a 37 micrómetros. Por otra parte, del análisis mineralógico realizado a la muestra se determinó que el mineral de apatita contenido en los relaves de hierro de la Compañía Minera del Pacífico, corresponde a una cloro apatita; 2) las pruebas preliminares de flotación, indicaron que se requiere utilizar silicato de sodio como dispersante de la ganga para alcanzar una buena recuperación y ley de fosfato en el concentrado, usando una dosificación de este reactivo en intervalos de $400 \mathrm{~g} / \mathrm{t}$, y 3) los mejores resultados obtenidos en la columna de flotación corresponden a las siguientes condiciones de operación: velocidad superficial de aire $1,4 \mathrm{~cm} / \mathrm{s}$, agua de lavado $0,41 \mathrm{~cm} / \mathrm{s}$, velocidad superficial de alimentación $1,4 \mathrm{~cm} / \mathrm{s}$ y altura de espuma $15 \mathrm{~cm}$, obteniendo una recuperación en peso de $17,6 \%$, una recuperación metalúrgica de $75,3 \%$ y una ley de $21,8 \%$ de $\mathrm{P}_{2} \mathrm{O}_{5}$ en el concentrado obtenido en una sola etapa de flotación.

\section{AGRADECIMIENTOS}

Los autores agradecen el apoyo financiero para el desarrollo del presente trabajo, aportado por el Fondo de Innovación para la Competitividad (FIC) de la Región de Atacama, por el proyecto "Desarrollo de tecnología innovadora para la producción de concentrados de minerales de valor económico contenidos en relaves de cobre de la Región de Atacama", a la Unidad de Geología de la Compañía de Acero del Pacífico y al Departamento de Ingeniería en Metalurgia de la Facultad de Ingeniería de la Universidad de Atacama, por el apoyo dado al mismo.

\section{REFERENCIAS}

Abdel-Zaher, M.A. Physical and thermal treatment of phosphate ores - an overview. International Journal of Mineral Processing 85, 59-84 (2008).

Al-Fariss, T.F., Abd El-Aleem, F.A. y Al-Qahtani, M.S. Beneficiation of saudi phosphate ores part II: The application of column flotation. $5^{\text {th }}$ International Conference on Heat Transfer, Fluid Mechanics and Thermodynamics Sun City, South Africa (2017).

Alsafasfeh, A. y Alagha $L$ Recovery of Phosphate Minerals from Plant Tailings, Using Direct Froth Flotation. Minerals 7(8), 145 https://doi.org/10.3390/min7080145_(2017).

Benzaazoua, M., Bussière, B., Demers, I., Aubertin, M., Fried, É., Blier, A. Integrated mine tailings management by combining environmental desulphurization and cemented paste backfill: application to mine Doyon, Quebec. Canada. Miner. Minerals Engineering 21, 330-340 (2008). 
Broadhurst, J.L., Kunene, M.C., von Blottnitz, H., Franzidis, J.P. Life cycle assessment of the desulfurisation flotation process to prevent acid rock drainage: A base metal case study. Miner. Eng. 76, 126-134 (2015).

Bruckard, W.J., McCallum, D.A. Treatment of sulphide tailings from base metal and gold operations - a source of saleable by-products and sustainable waste management, World Gold Congress, Cairns, Australia, 22-24 October 2007, 85-91 (2007).

Choi, W.H., Lee, S.R., Park, J.Y. Cement based solidification/stabilization of arsenic-contaminated mine tailings. Waste Manage. 29, 1766-1771 (2009).

Durance, M.V., Botan, P., Cailleau, A., 2010. Reprocessing of a French Guyana operation's tailings using flotation for gold concentration. Minerals \&Metallurgical Processing 27, 55-63. (2010).

Edraki, M., Baumgartl, T., Manlapig, E., Bradshaw, D., Franks, D. M., Moran, C. J. Designing mine tailings for better environmental, social and economic outcomes: a review of alternative approaches. J. Clean. Prod. 84, 411-420 (2014).

Eisele, T. C. and Kawatra, S. K, "Phosphate Flotation using a Horizontally Baffled Column", Chapter 17, Beneficiation of Phosphates, (eds. Zhang, El-Shall and Wiegel), Pub. Society of Mining, Metallurgy and Exploration, Littleton, CO., 211214. (1999).

Falagán, C., Grail, B.M., Johnson, D.B. New approaches for extracting and recovering metals from mine tailings. Miner. Eng. 106, 71-78 (2017).

Grauch, R.I., Verplanck, P.L., Seeger, C.M., Budahn, J.R., and Van Gosen, B.S. Chemistry of selected core samples, concentrate, tailings, and tailings pond waters-Pea Ridge iron (-lanthanide-gold) deposit, Washington County, Missouri: U.S. Geological Survey Open-File Report 2010-1080. (disponible en http://pubs.usgs.gov/ of/2010/1080/.) (2010).

Herrera, F. Estudio Geológico de relave de minas El Romeral, análisis de proceso Geo metalúrgicos para la recuperación de especies de especies mineralógicas valiosas con énfasis en apatito. Memoria para optar al título de geólogo. (2018).

Hunter, T.N., Pugh, R.J., Franks, G.V. and Jameson, G.J. The Role of Particle in Stabilising Foams and Emulsions. Advances in Colloid and Interface Science, 137, 57-81. http://dx.doi.org/10.1016/j.cis.2007.07.007 (2008).

Laskowski J.S. Frothing in flotation. Gordon and Brach science publishers; (1989).

Leistner, T., Embrechts, M., Leißner, T., Chehreh Chelgani, S., Osbahr, I., Möckel, R., Peuker, U.A., Rudolph, M. A study of the reprocessing of fine and ultrafine cassiterite from gravity tailing residues by using various flotation techniques. Miner. (2016).

Liang, J. and Elias, D. Seismic Evaluation of Tailing Storage Facility. Australian Earthquake Engineering Society 2010 Conference, Perth, Western Australia (2010).

Mbamba, C.K., Harrison, S.T.L., Franzidis, J.P., Broadhurst, J.L. Mitigating acid rock drainage risks while recovering lowsulfur coal from ultrafine colliery wastes using froth flotation. Miner. 29, 13-21 (2012).

Memoria Anual CAP, 2017. Consultado 12/09/2018 http://www.cap.cl/cap/ (2017).

Oliva, J. Recuperación de apatita mediante flotación de los relaves de hierro de la Compañía Minera del Pacífico. Trabajo para obtener el Título de Ingeniero Civil en Metalurgia, Universidad de Atacama. (2018)

Oliveira, M.S., Santana, R.C., Ataíde, C.H., Barrozo, M.A.S. Recovery of apatite from flotation tailings. Sep. Purif. Technol. 79, 79-84 (2011).

Robinstein, J.B. Column Flotation, Processes, Design and Practices, Gordon and Beach Science Publishers, Basilea, Suiza (1995).

Schulze, H. J. New theoretical and experimental investigation on stability of bubble/particle aggregates in flotation - A theory on the upper particle size of flotability. International Journal of Mineral Processing, 4, 241 - 259. (1977).

SERNAGEOMIN. Relaves mineros http://www.sernageomin.cl/ Consultado 12 de marzo (2017).

Tariq F. Al-Fariss, Farag A. Abd El-Aleem, Khaled A. El-Nagdy. Beneficiation of Saudi phosphate ores by column flotation technology. Journal of King Saud University - Engineering Sciences 25, 113-117 (2013).

Valderrama, L. Santander, M. Paiva M. and Rubio, J.. Modified-three-product column (3PC) flotation of copper-gold particles in a rougher feed and tailings. Minerals Engineering, 24, No. 13, (2011).

Valderrama, L., Rojas, S. Zazzali, B. Olguín, D. Detección de los Elementos de Tierras Raras contenidos en Relaves y Ripios de la Planta (Osvaldo Martínez) de la Empresa Nacional de Minería. Revista de Ingeniería de la Facultad de Ingeniería de la Universidad de Atacama, N 16, 27- 32 (2003).

Von Holt, S.T., Franzidis, J.P. Column flotation of South Africa cooking coal. Journal of Coal Preparation 14, 147-166 (1994).

Wang, C., Harbottle, D., Liu, Q., Xu, Z. Current state of fine mineral tailings treatment: a critical review on theory and practice. Miner. Eng. 58, 113-131 (2014).

Wolkersdorfer, C., Bowell, R. Contemporary reviews of mine water studies in Europe. Mine Water Environ. 23 (4), 162182. (2014). 